\title{
Atualização Colaborativa do Modelo de Processos de Negócio
}

\author{
Nuno Castela 1,2, Paulo Dias 1, Marielba Zacarias 2,3, José Tribolet 2,4 \\ ncastela@ipcb.pt, paulo.dias@ipcb.pt, mzacaria@ualg.pt, jose.tribolet@inesc.pt \\ ${ }^{1}$ Escola Superior de Tecnologia do Instituto Politécnico de Castelo Branco, Av. Do Empresário, 600o-767 \\ Castelo Branco, Portugal \\ ${ }^{2}$ Center for Organizational Design and Engineering, INESC INOVAÇÃO, Rua Alves Redol, ${ }^{\circ}$ 9, 1000-029 \\ Lisboa, Portugal \\ 3 Research Center for Spatial and Organizational Dynamics, University of Algarve, Campus de Gambelas, \\ 8005-139 Faro, Portugal \\ 4 Dep. of Computer Science and Engineering, IST, University of Lisbon, Campus Alameda, Av. Rovisco Pais, \\ 1, 1049-001 Lisbon, Portugal
}

DOI: 10.4304/risti.12.33-47

\begin{abstract}
Resumo: O modelo de processos de negócio as-is pode ajudar a melhorar a consciência organizacional através da partilha de conhecimento dos atores organizacionais. No entanto, a dificuldade de atualização do modelo e o seu padrão típico de utilização têm evitado a sua transformação num repositório do conhecimento organizacional que suporte as atividades diárias das organizações. Para ultrapassar este problema definiu-se um método colaborativo para atualizar o modelo de processos de negócio, utilizando o mecanismo de anotação para criar contextos de interação que promovam a explicitação e comunicação do conhecimento e a discussão dos processos. Desenvolveu-se uma ferramenta de suporte ao método colaborativo de atualização que foi utilizada em casos de estudo. A abordagem proposta permitiu demonstrar que os atores organizacionais, desde que providos dum método adequado e uma ferramenta de suporte, podem atualizar ativamente o modelo de processos de negócio, comparando as atividades modeladas com as atividades que são efetivamente executadas.
\end{abstract}

Palavras-chave: Modelação Colaborativa, Atualização Colaborativa de Processos de Negócio, Conhecimento Organizacional.

\section{Business Process Model Collaborative Updating}

Abstract: Business process modeling helps improving organizational selfawareness by sharing organizational knowledge. It is acknowledged that the maintenance of the models is a difficult task and that in most of the time companies build models to use in specific projects occurring at a given time, what have prevented its transformation into a repository of organizational knowledge to support the daily activities of organizations. The present work defines an "as-is" 
model continuous updating method that uses the annotation mechanism to create interaction contexts and to enable business actors to make explicit and communicate their knowledge about processes and their own work, and also to discuss the existing process representations. A prototype tool has been developed and was used in several case studies. This approach has demonstrated that organizational actors, since provided with a method and supporting tool, can act as active updaters of the process model by comparing modeled with actually executed activities.

Keywords: Collaborative Modeling, Business Process Collaborative Updating, Organizational Knowledge.

\section{Introdução}

O modelo empresarial representa vários aspetos, vistas ou perspetivas das organizações. As vistas mais comuns incluem a vista estratégica ou organizacional, a vista de processos, a vista de informação, a vista aplicacional e a vista tecnológica (Schekkerman, 2004). A vista de processos de negócio é uma das mais importantes do modelo empresarial. Esta vista mostra os fluxos de atividades, assim como as suas entradas e saídas. Caso o modelo de processos de negócio constituísse uma representação fidedigna e atual dos processos das organizações, poderia ser usado permanentemente como base de apoio para as mais variadas tarefas operacionais e de gestão, já que permitiria tirar partido das suas capacidades de captura, representação e distribuição do conhecimento organizacional. No entanto, o modelo de processo de negócio tem sido usado principalmente para apoiar tarefas organizacionais esporádicas no tempo (projetos de reengenharia de processos, arquiteturas de sistemas de informação, captura de requisitos para projetos de SI/TI, etc.), por oposição a constituir uma base de apoio às tarefas do dia-a-dia organizacional que sirva de repositório do conhecimento organizacional com utilização universal.

Esta utilização descontínua do modelo de processos de negócio não deriva da falta de reconhecimento das suas potencialidades, mas sim da dificuldade em mantê-lo atualizado e alinhado com a realidade organizacional.

A engenharia organizacional junta conceitos, métodos e tecnologias que permitem compreender, modelar, desenvolver e analisar todos os aspetos do negócio em mudança através do foco na compreensão dos relacionamentos e dependências entre a estratégia, os processos e os sistemas de informação que os suportam (Tribolet, Winter \& Caetano, 2005). A investigação nesta área tem dado passos significativos na última década no sentido da definição quer dos artefactos e linguagens de modelação, quer das vistas necessárias à sua adequação aos atores organizacionais que permitirá a sua utilização numa base contínua no tempo. Esta investigação tem sido enriquecida com o cruzamento de áreas do saber que vão desde a engenharia às ciências sociais, permitindo uma visão clara do que são as organizações, como funcionam e como aprendem.

O presente trabalho propõe um método colaborativo para a atualização dinâmica do modelo de processos de negócio, confiando aos atores organizacionais a monitorização contínua das atividades que desempenham e a consequente proposta de atualizações à sua representação. A atualização do modelo de processos de negócio é, no método 
proposto, implementada através do mecanismo de anotação do modelo, que permite estabelecer uma conversação entre os atores envolvidos sobre a representação da própria organização. As propostas de atualização são revistas e avaliadas e, dependendo do resultado da avaliação, podem levar à criação de novas versões do modelo de processos de negócio. No âmbito desta investigação desenvolveu-se uma ferramenta que implementa o modelo proposto e que foi testada em vários contextos organizacionais.

$\mathrm{Na}$ secção seguinte deste documento apresenta-se o estado da arte que suportou a definição do método de atualização dinâmica do modelo de processos de negócio as-is, cujas ideias chave são apresentadas na secção 3. Na secção 4 são apresentados os requisitos gerais da ferramenta colaborativa de suporte ao método definido. Finalmente, na secção 5 são enunciados os resultados dos casos de estudo realizados, e na secção 6 as conclusões e o trabalho futuro.

\section{Estado da Arte}

Partindo da seguinte definição de organização (Magalhães \& Tribolet, 2007): “Uma organização é o resultado das ações dos seus atores humanos e não humanos organizados em sistemas sociotécnicos que se autorrealizam através das ações e interações dos seus componentes, e que têm como fator diferenciador em relação à restante criação humana o facto de elas próprias envolverem na sua constituição agentes humanos", o conceito de consciência organizacional aparece naturalmente como o resultado do cruzamento complexo das várias consciências - individuais, de grupo, funcionais e processuais - que guiam a ação corrente nas organizações, dependendo dos contextos invocados. Zacarias, Magalhães, Caetano, Pinto e Tribolet (2008) concretizam esta noção indicando que a consciência organizacional é a capacidade que as organizações possuem para responder em qualquer momento perguntas específicas sobre as suas atividades, recursos e atores.

Os processos e as atividades são os elementos que contêm toda a informação sobre como, quando e quem faz fluir o trabalho (Magalhães \& Tribolet, 2007). As primitivas, a sintaxe e a semântica do modelo de processos de negócio devem permitir aos atores organizacionais verificarem os processos de negócio, a partir da realidade contínua da sua ação. Isto implica que a base de representação tenha que estar nas atividades concretas desenvolvidas, porque esta é a única base realmente verificável e compreensível pelos atores organizacionais (Tribolet, 2005). O Modelo de processos de negócio, integrado na Arquitetura Empresarial, tem um papel fundamental na representação do conhecimento e consciência organizacional, e na possibilidade de servir como objeto de conversação para incorporar mais conhecimento de forma iterativa e incremental. Este papel deve-o maioritariamente às suas características intrínsecas de representação dinâmica das ações e interações organizacionais centradas na execução dos processos como sequências orquestradas das atividades.

A modelação sempre esteve no centro das atividades e metodologias de gestão por processos (BPM), pois os modelos de processos têm sido utilizados na melhoria, reengenharia, certificação e iniciativas de implementação de tecnologias de informação nas organizações (Adamides \& Karacapilidis, 2006). Portanto, reconhece-se que tem havido muita discussão sobre as características e adequação dos diferentes 
formalismos de modelação mas tem sido dada pouca atenção ao próprio processo de modelação como um processo sociocognitivo.

O modelo de processos de negócio de uma organização representa a forma como os processos são executados na vida real. Apesar de modelos de processos serem considerados como tendo um grande potencial para a partilha do conhecimento organizacional o seu potencial é geralmente negligenciado, principalmente porque são usados por técnicos, analistas, programadores e gestores, que usam sistemas de BPM para gerir processos de negócios (Bruno, Dengler, Jennings, Khalaf, Nurcan, Prilla, Sarini, Schmidt, \& Silva, 2011). Os modelos de processos de negócio são muitas vezes construídos e depois descartados porque a maioria dos objetivos organizacionais que levam à sua construção permite que o modelo seja considerado um artefacto técnico que é usado para suportar tarefas isoladas no tempo (Castela, Dias, Zacarias \& Tribolet, 2012). Este fenómeno também é agravado pela natureza dinâmica das organizações, que mudam muito depressa, sem que as alterações sejam feitas ao modelo criado. Como resultado, os participantes nos processo ou novos funcionários, que precisam conhecer ou dar feedback sobre os processos de negócios, não têm acesso ao conhecimento dos modelos de processos (Bruno et al., 2011).

Rittgen (2007) refere que apesar da literatura sobre modelação ser abundante, a maioria descreve a utilização da notação de uma forma prescritiva e não descritiva, pois não são referidos os problemas mais comuns que as pessoas sentem durante o processo de modelação. Rittgen (2007) considera a modelação como uma conversação do tipo negociação e estabeleceu um padrão de negociação no contexto da modelação colaborativa de processos de negócio, definindo uma série de atividades que guiam e disciplinam a negociação.

A modelação colaborativa pode aumentar a produtividade e qualidade da modelação contribuindo para a construção de acordos e de um sentimento de propriedade entre os stakeholders (Ssebuggwawo, Hoppenbrouwers, \& Proper 2009). A fim de chegar a um consenso ou acordo, os modeladores devem comprometer-se a trabalhar em equipa e respeitar os conhecimentos coletivos, convenções e decisões. A estratégia de comunicação define as metas e regras (explícita ou implicitamente) para um diálogo de conversação em que os modeladores possam propor e discutir (negociar) diferentes posições. Esta comunicação pode resultar em acordo ou desacordo e levar à aceitação ou rejeição das ideias propostas.

O processo de modelação colaborativa pode ser suportado por ferramentas de modelação colaborativas, que implementem a componente de negociação que facilite a estruturação de argumentos e decisões relacionados com as escolhas de modelação (Rittgen, 2007).

Gonzalez e Framinan (2010) identificaram um conjunto de características necessárias às ferramentas colaborativas de modelação de processos de negócios e analisaram um conjunto de ferramentas comerciais para verificar se as características identificadas estão implementadas. Em geral a maioria das ferramentas analisadas proporciona características colaborativas, mas muitas das funcionalidades não estão presentes a não ser que, em alguns casos, todos os módulos que possuem sejam comprados, ou noutros casos, sejam comprados módulos de outros fornecedores. Os requisitos das ferramentas de modelação colaborativa de processos de negócios podem ser 
classificados de acordo com a matriz espacial-temporal de groupware (Ellis, Gibbs, \& Rein, 1991), pois a interação pode acontecer ao mesmo tempo (síncrono) ou em tempos diferentes (assíncrono) e os participantes na interação pode ser no mesmo local ou locais diferentes.

\section{Método de Atualização Colaborativa de Processos de Negócio}

A ideia a explorar para definição do Método de Atualização Colaborativa de Processos (MACoP) teve por base a análise de desalinhamentos entre o modelo distribuído e a realidade. Esta análise de desalinhamento utilizará as anotações como mecanismo de captura das atualizações propostas pelos agentes organizacionais.

A utilização no decurso deste trabalho do conceito de anotação, baseou-se no trabalho de Becker-Kornstaedt e Reinert (2002) que aplicaram este conceito como mecanismo para capturar as razões para as alterações feitas no âmbito de projetos de desenvolvimento de software a partir do conhecimento implícito das equipas de desenvolvimento.

As anotações devem capturar as atividades, os recursos (entidades) e o contexto envolvido (fluxos). A melhoria contínua dos processos requer que a experiência seja capturada para que seja continuamente incorporada nos processos de negócio e continuamente retratada no modelo as-is.

A captura e armazenamento sistemático no contexto onde a experiência foi capturada têm três grandes benefícios (Becker-Kornstaedt \& Reinert, 2002):

- A experiência ganha pode tornar-se explícita.

- A experiência pode ser integrada na própria descrição do processo.

- A análise da experiência pode ser reutilizada noutros processos para melhoria dos processos.

O MACoP é executado pelas pessoas (atores organizacionais) que existem no plano operacional e que partilham uma representação comum dos processos de negócio.

As anotações servem para fazer propostas de alteração ao modelo apresentado de forma a alinhá-lo com a realidade percebida por cada ator organizacional. Estas propostas que têm como objetivo fazer a manutenção corretiva do modelo de processos de negócio, podem ter dois objetivos: corrigir o modelo apresentado ou aumentar o seu detalhe. Após a realização de uma anotação sobre um elemento de modelação (que dependerá da granularidade ou nível do modelo apresentado) poderá existir uma fase de negociação com os atores que eventualmente partilham o mesmo contexto de ação. Esta discussão será realizada por todos os interessados no elemento anotado com vista à clarificação do propósito da anotação original. Todos os atores envolvidos nesta revisão deverão declarar a concordância ou discordância com a anotação feita ao elemento do modelo $\mathrm{Na}$ sequência da revisão da anotação, a anotação deverá ser avaliada pelos atores habilitados para o fazer, e que possuem um nível de responsabilidade sobre as atividades executadas ou sobre os atores organizacionais envolvidos. Caso a avaliação da anotação (e eventuais revisões) resulte numa 
aprovação, as alterações solicitadas na anotação devem ser incorporadas na nova versão do modelo de processos de negócio.

A figura 1 mostra a estrutura que (1) identifica as fases do MACoP (lado direito da figura) e (2) mostra o padrão de negociação associado às fases de Revisão e Avaliação, despoletados pela fase de anotação (lado esquerdo da figura). Este padrão de negociação é baseado num padrão negociação que considera que a modelação como uma conversação do tipo negociação (Rittgen, 2007).

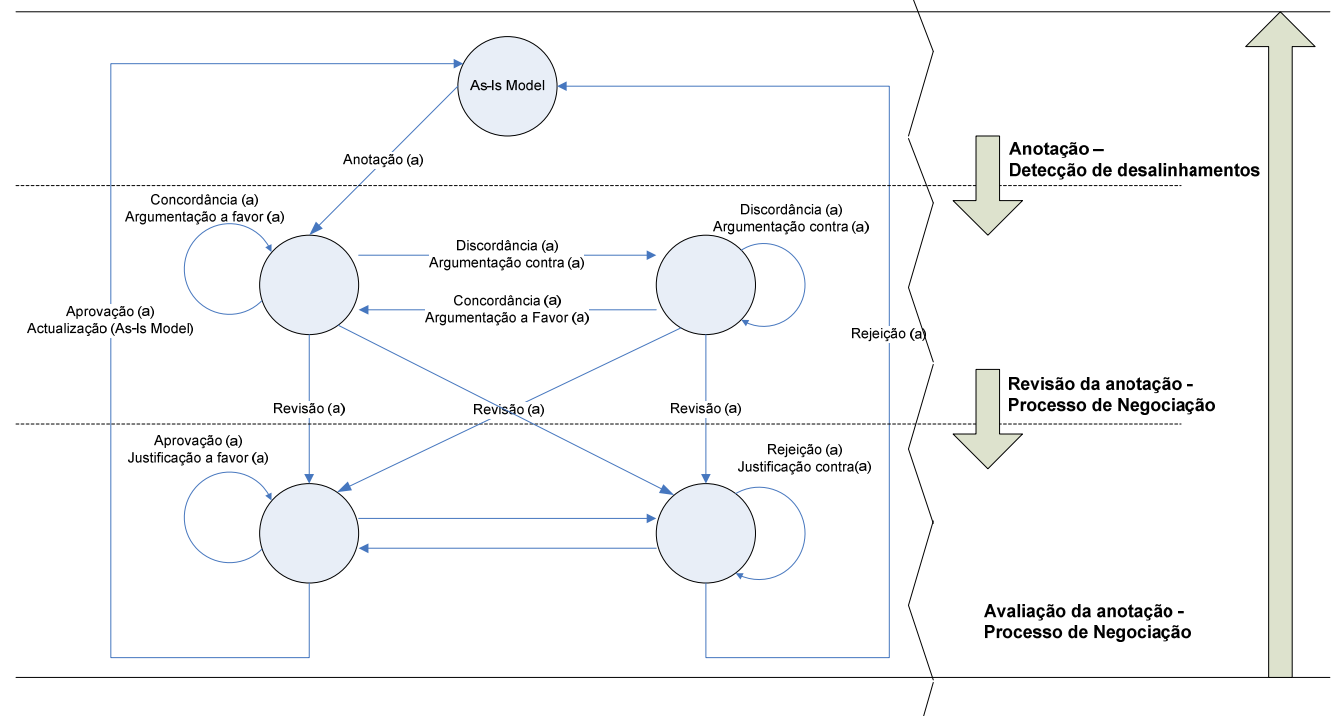

Figura 1 - Padrão de Negociação e Fases do Método MACoP

O contexto de interação considerado é o do próprio processo de negócio modelado em BPMN, já que permitirá a visualização do modelo no qual os atores executantes participam e que servirá para fazerem anotações ao nível do próprio processo como um todo ou ao nível dos elementos que compõem o processo em BPMN (atividades, entidades informacionais, fluxos e unidades organizacionais).

Neste contexto de interação, os atores organizacionais podem ser, no plano operacional executantes de atividades ou donos dos processos. No plano de atualização colaborativa podem tomar os papéis de anotador, revisor ou avaliador, se, respetivamente, fizerem anotações a qualquer elemento de modelação do processo, se fizerem revisões a qualquer anotação feita ao processo ou se aprovarem ou rejeitarem qualquer anotação. Os executantes do plano operacional podem tomar o papel de anotador ou revisor, enquanto apenas o dono do processo pode tomar o papel de avaliador, para além de poder ser anotador ou revisor.

\section{Ferramenta MAPA}

Para suportar o Método de Atualização Colaborativa de Processos de Negócio, desenvolveu-se um protótipo de ferramenta Web de groupware a que se chamou 
MAPA (de Modelação e Atualização de Processos e Atividades) com os seguintes requisitos gerais:

- Funções de edição de anotações: os executantes dos processos necessitam de suporte para fazer anotações imediatas no contexto onde ocorre a experiência. Foi criado um sistema de anotações para a criação, modificação e apagamento de anotações pelos utilizadores.

- Níveis de granularidade diferentes: deve ser possível anotar qualquer objeto no modelo de processos, i.e., cada processo, atividade, papel, recurso, relação e cada atributo de cada objeto.

- Capacidade de distribuição seletiva de diagramas e elementos de modelação: os utilizadores apenas acedem à informação que lhes diz respeito, dependendo do papel invocado (executante, dono de processo e responsável de unidade organizacional).

- Direitos de acesso: para proteger os autores das anotações, devem ser suportados níveis diferentes de direitos de acesso dependendo dos papéis atribuídos a cada ator organizacional.

- Capacidade de guardar todo o histórico de modelos e respetivas anotações, revisões e avaliações.

- Mapeamento de anotações às entidades: para cada anotação é fundamental saber-se a que elemento de modelação diz respeito.

- Suporte à negociação, pois é necessário que a ferramenta dê suporte às fases que envolvem negociação entre os atores organizacionais (nomeadamente na revisão e avaliação das anotações).

- Mecanismos de notificação: para alertar os atores organizacionais das eventuais ações de revisão e/ou avaliações que terão que executar e de atualizações que tenham sido feitas aos diagramas.

- Capacidades de desenho: para que os atores anotadores possam fazer anotações gráficas com propostas de alterações aos modelos e para que os atores avaliadores possam alterar o modelo diretamente caso a(s) proposta(s) de alteração tenham sido aprovadas.

A vista principal de interação com os utilizadores da ferramenta MAPA (figura 2) é o modelo de processos de negócio modelado em BPMN, que permitirá a visualização do modelo no qual os atores executantes participam e que servirá para fazerem anotações ao nível do próprio processo como um todo ou ao nível dos elementos que compõem o processo em BPMN (atividades, Entidades Informacionais, Fluxos e lanes). 


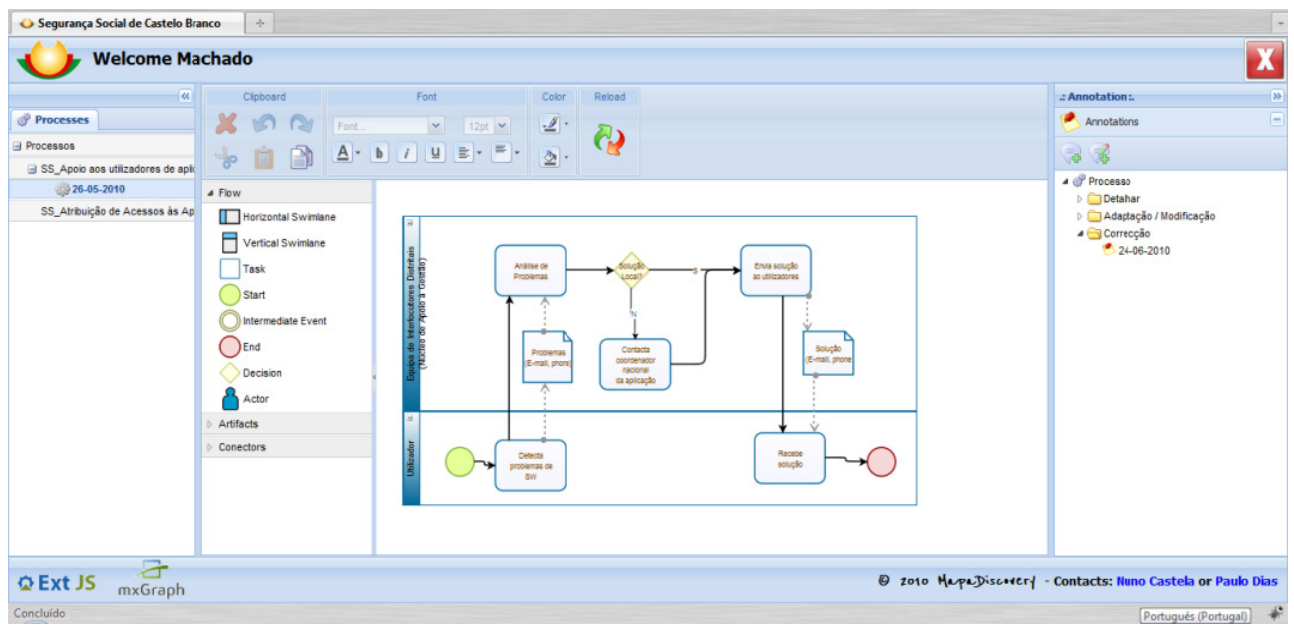

Figura 2 - Ecrã principal da ferramenta MAPA

A ferramenta MAPA guarda os modelos de processos de negócio as-is numa base de dados, ficando a garantia de integridade, quer do modelo as-is, quer das correspondentes anotações (e eventuais revisões e aprovações) da responsabilidade do modelo relacional definido e implementado num sistema de gestão de bases de dados. O histórico dos modelos anteriores e das anotações, revisões e avaliações que levaram à sua evolução ficam também armazenados no sistema de gestão de base de dados.

\section{Casos de Estudo}

Foram criados quatro casos de estudo para avaliar o MACoP (figura 3).

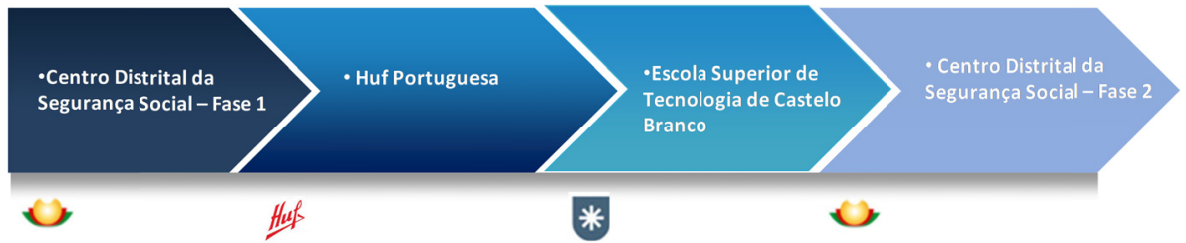

Figura 3 - Sequência de implementação de estudos de caso

O primeiro estudo de caso foi realizado no Centro Distrital de Segurança Social (CDSS) de Castelo Branco, com uma versão preliminar da ferramenta MAPA (fase 1). O segundo caso de estudo foi realizado na Huf Portuguesa, uma empresa industrial do setor automóvel, onde a ferramenta MAPA foi usada inicialmente para modelar os processos de negócios a partir de um modelo ultrapassado existente, de forma colaborativa. Quando este modelo foi terminado, pôde-se aplicar o MACoP para manter os processos atualizados. O terceiro caso de estudo foi desenvolvido na Escola Superior de Tecnologia de Castelo Branco para atualização contínua dos processos de negócio dos Serviços Académicos. O caso de estudo seguinte, decorreu novamente no CDSS (fase 2), e usou a ferramenta MAPA em dois dos processos de negócio já 
modelados na fase 1 e 17 novos processos de negócio. Foram modelados 2 processos da Equipa de Interlocutores distritais, 12 processos da Equipa de Gestão Financeira e 5 processos da Equipa de Administração e Património do CDSS.

Para ilustrar a dinâmica de interação fornecidos pela ferramenta, apresentam-se a seguir alguns exemplos de anotações, revisões e posteriores aprovações feitas no contexto de um processo de negócio do CDSS. A Figura 4 mostra o diagrama inicial dlo processo de negócio "Compras e Gestão de Materiais" modelado na ferramenta MAPA. Este processo mostra a interação no Centro Distrital de Segurança Social, entre a Equipa de Aprovisionamento e Património, a Equipa de Gestão Financeira, os Serviços Centrais e os fornecedores, necessários para gerir a aquisição de serviços e equipamentos para o CDSS.

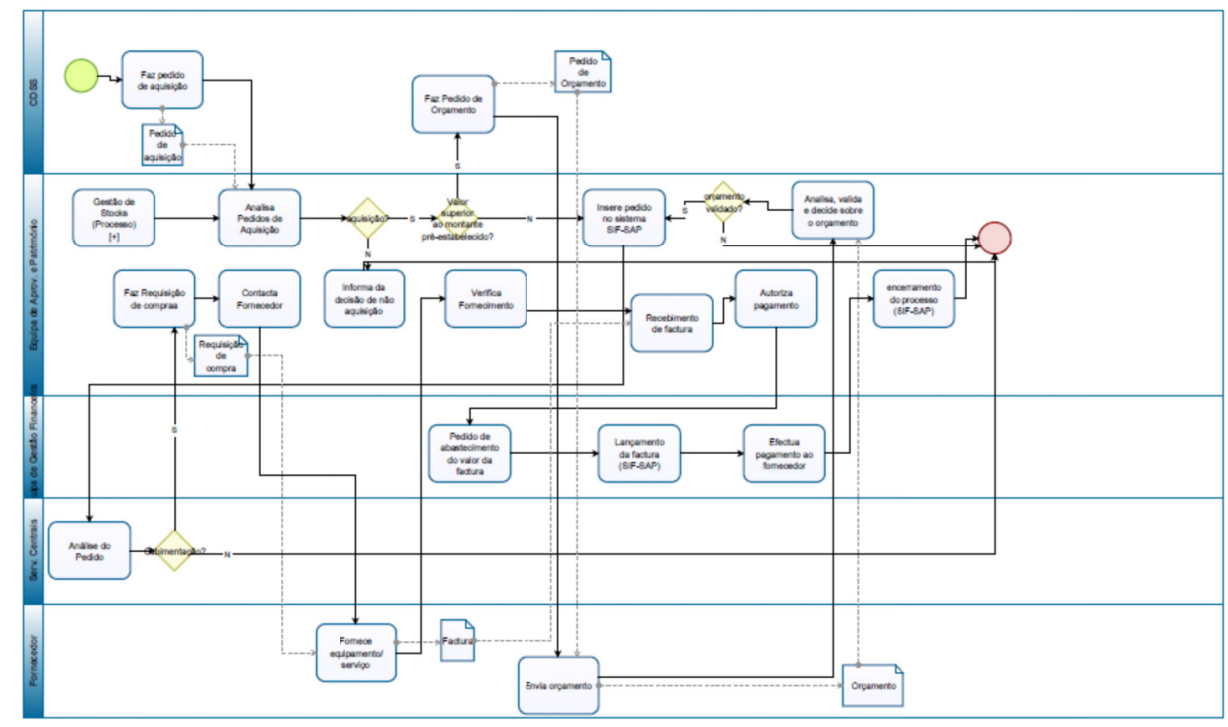

Figura 4 - Primeira versão do processo de negócio "Compras e Gestão de Materiais"

A figura 5 mostra a anotação (textual) feita pelo ator organizacional Octávio Gil e os correspondentes comentários feitos por dois colegas que participam no mesmo processo de negócio que concordaram com a anotação feita. Na mesma figura também podemos ver a avaliação da anotação (aprovação) feita a anotação pela responsável da equipa, Sara Soares. 


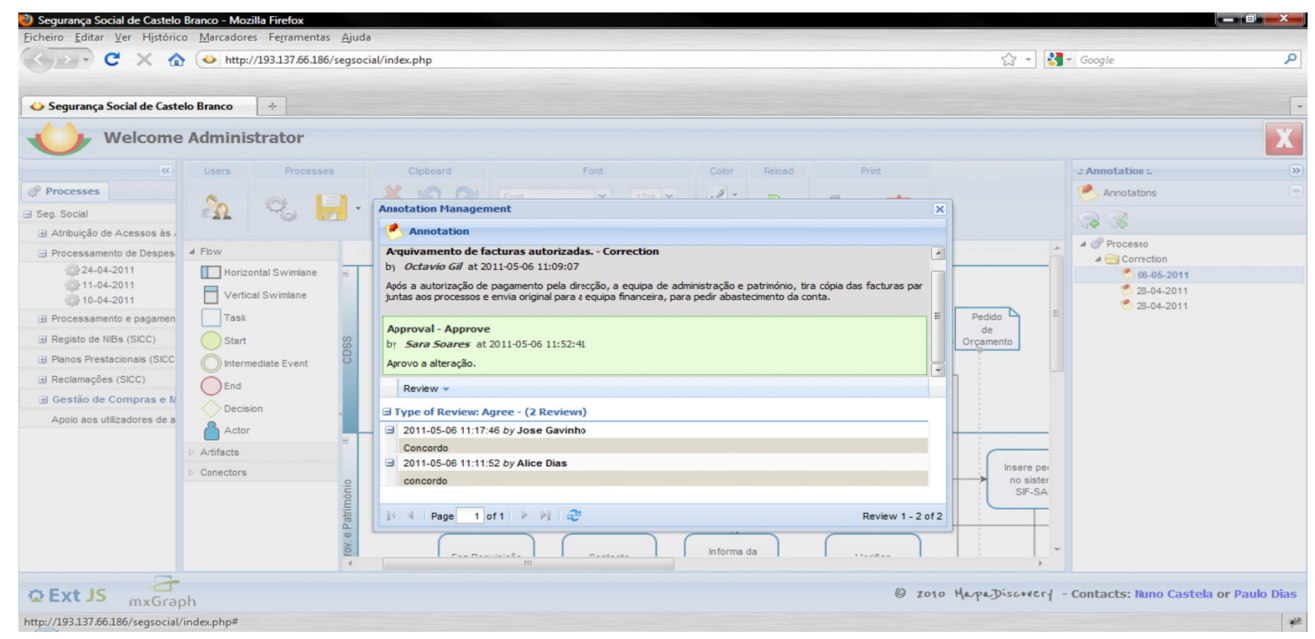

Figura 5 - Anotação textual, comentários e avaliação feita para o processo de negócio

A Figura 6 mostra uma anotação gráfica feita no âmbito da mesma versão do processo "Compras e Gestão de Materiais" por Alice Dias. Podem também ser vistos os comentários e a avaliação feita para essa anotação, a qual, além da descrição textual, contém uma descrição do gráfico mostrado na figura 7.

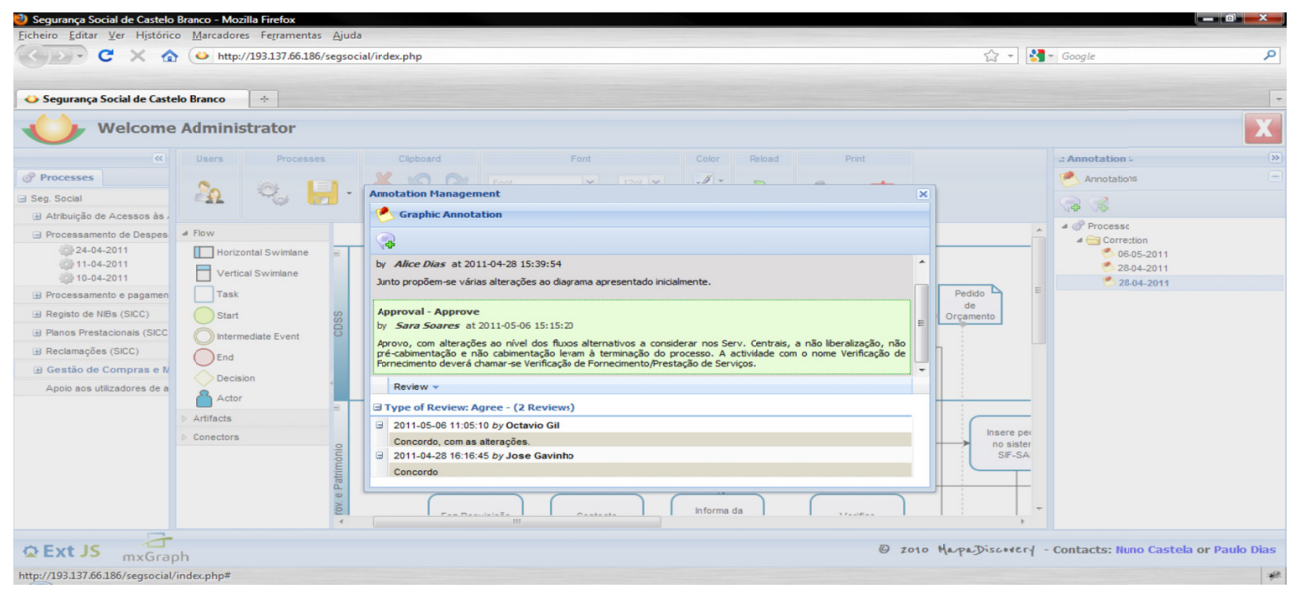

Figura 6 - Anotação gráfica com comentários e avaliação 


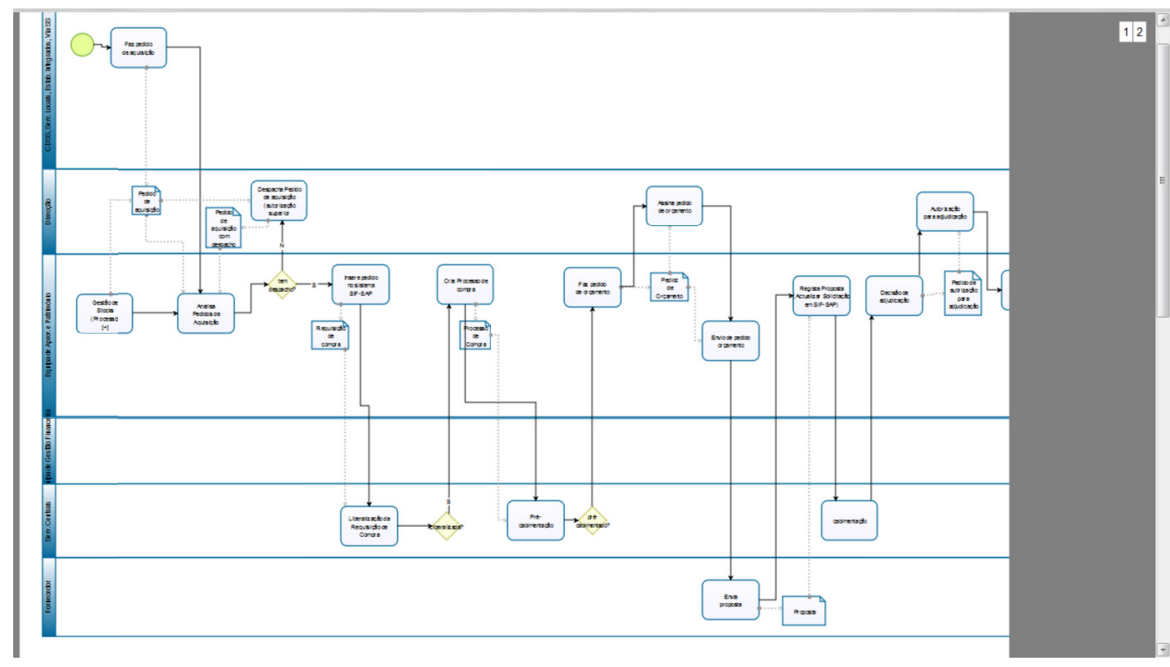

Figura 7 - Propostas de mudança gráfica da anotação feita

A Figura 8 mostra a nova versão do processo "Compras e Gestão de Materiais", modelado na ferramenta MAPA pela responsável da equipa, após a consideração de todas as anotações e revisões feitas pelos atores executantes do processo.

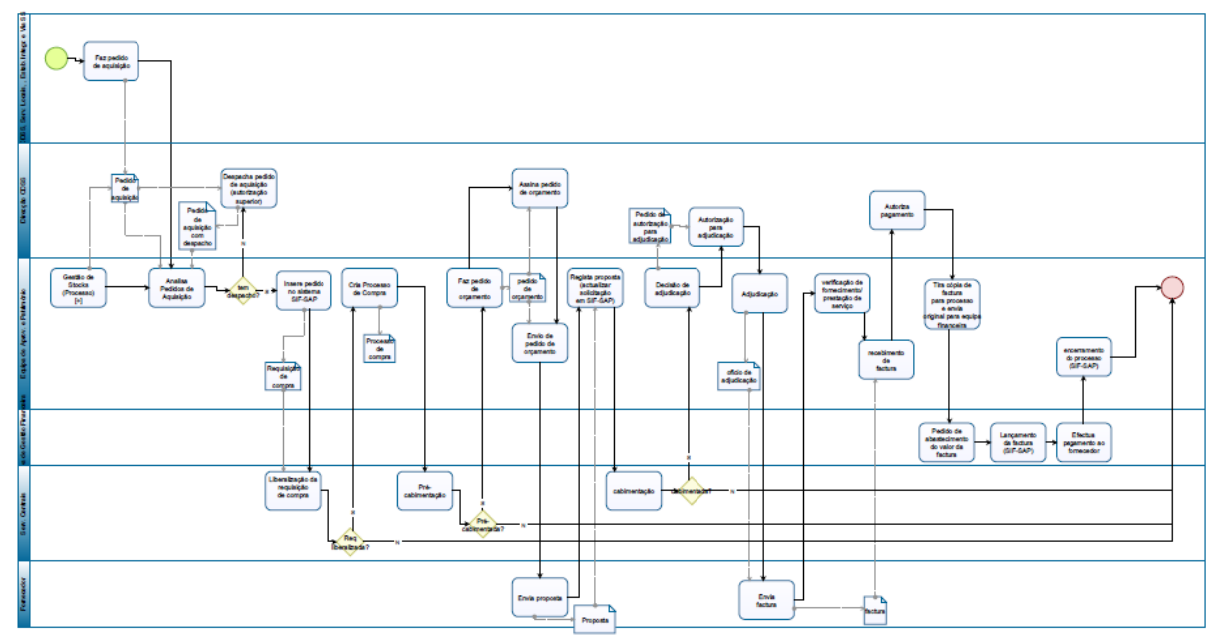

Figura 8 - Nova versão de Compras e Gestão de Materiais " processo de negócio

A Tabela 1 mostra o resumo dos resultados obtidos a nível global nos dois casos de estudo desenvolvidos onde a ferramenta MAPA foi usada para manter o modelo de processo de negócio as-is, promovendo a sua atualização dinâmica e distribuída: Segurança Social (fase 2) e Escola Superior de Tecnologia de Castelo Branco.

A tabela 1 descarta os resultados da fase 1 do caso de estudo da Segurança Social, porque nesse caso foi usada uma versão da ferramenta MAPA muito incipiente. 
Descarta igualmente os resultados da utilização na Huf Portuguesa, porque neste caso de estudo a ferramenta MAPA foi usada maioritariamente para traduzir, de forma colaborativa, o antigo modelo de processos de negócios para um novo modelo usando BPMN. Os resultados apresentados referem-se a realização de 2 ciclos completos do MACop.

Tabela 1 - Resumo dos resultados de estudos de caso

\begin{tabular}{|c|c|c|}
\hline \multirow{2}{*}{$\frac{\text { MACoP (2 ciclos) }}{\text { Modelação }}$} & \multicolumn{2}{|c|}{ Sumário dos resultados (CDSS - fase 2 + ESTCB) } \\
\hline & Numero de processos & 21 \\
\hline \multirow{7}{*}{ Anotação } & Numero de processos anotados & 16 \\
\hline & Numero de anotações feitas & 36 \\
\hline & Percentagem de processos anotados & $76,2 \%$ \\
\hline & Numero de anotações textuais & 22 \\
\hline & Percentagem de anotações textuais & $61,1 \%$ \\
\hline & Anotações gráficas & 14 \\
\hline & Percentagem de anotações gráficas & $38,9 \%$ \\
\hline \multirow{4}{*}{ Revisão } & Numero de revises & 45 \\
\hline & Percentagem de anotações revistas & $69,4 \%$ \\
\hline & Revisões do tipo "I agree" & 44 \\
\hline & Revisões do tipo "I do not agree" & 1 \\
\hline \multirow{3}{*}{ Avaliação } & Numero de avaliações & 29 \\
\hline & Numero de anotações avaliadas & $80,6 \%$ \\
\hline & Avaliações do tipo "approval” & 29 \\
\hline \multirow[b]{2}{*}{ Modelação } & Processos atualizados (por ciclo de MACop) & $16+2$ \\
\hline & Percentagem de processos atualizados (no $1^{\circ}$ ciclo) & 76,2 \\
\hline
\end{tabular}

Das 36 anotações feitas, 52,8 \% foram do tipo "correção", 22,2 \% do tipo "adaptação" e 25,0 \% do tipo "aumento de detalhe". Estes resultados estão diretamente ligados com a validação das primeiras versões do modelo de processo de negócio modelados com a ferramenta MAPA, já que anteriormente não existia qualquer modelo de processos de negócio nas duas organizações envolvidas. Também relevante é a percentagem relativamente elevada de anotações gráficas realizadas: $38,4 \%$.

A partir das respostas às entrevistas realizadas para validar os resultados, 93\% dos atores organizacionais fizeram anotações textuais e 40\% usaram as anotações gráficas. Pudemos constatar que todos os atores consideraram importante a sua participação na discussão do processo, assim como o suporte dado pela ferramenta MAPA. 94\% dos atores organizacionais consideram importante a possibilidade de discussão dos processos de negócio em que participam, através das revisões às anotações feitas aos processos.

\section{Conclusões e Trabalho Futuro}

O MACoP e a ferramenta MAPA estão neste momento a ser utilizados em duas organizações públicas (Escola Superior de Tecnologia do Instituto Politécnico de Castelo Branco e Centro Distrital de Segurança Social de Castelo Branco) e numa organização privada multinacional do sector automóvel (Huf Portuguesa), no âmbito dos casos de estudo descritos no capítulo 5. Para além destas organizações, o MACoP e 
a ferramenta MAPA estão também a ser utilizadas noutras organizações de forma autónoma (Universidade do Algarve e Teatro Viriato).

Com os resultados disponíveis verificou-se que os atores organizacionais anotaram correções ao modelo relacionadas com a validação dos diagramas produzidos, permitindo no entanto envolver os atores relacionados diretamente (executantes, donos de processos e chefes de unidades organizacionais) na validação (e consequentemente atualização) do modelo, alinhando-o com a realidade de forma interativa e partilhada. Pela realidade observada nos casos de estudo, as anotações e as suas extensões (revisões e avaliações) preenchem o requisito de serem mecanismos adequados a apoiarem a conversação entre os atores e a sua representação.

O MACoP é importante no incremento da consciência individual, pois ao fornecer aos atores representações explícitas, estes ficam com uma melhor noção daquilo que fazem e do contexto envolvente. É também importante por incrementar a consciência dos grupos criados ao nível dos processos e das atividades. A consciência organizacional ganhou com o contributo e explicitação do conhecimento individual e de grupo, através da criação do histórico de evolução dos processos (versões dos diagramas dos processos), que incluem o histórico de anotações (e respetiva negociação/discussão).

O MACoP é uma mais-valia para as organizações pois permite maximizar a aproximação entre a "realidade" operacional e a sua representação, envolvendo, após a deteção dos desalinhamentos, negociações para revisão das anotações (revisão pelos pares - executantes que partilham os contextos operacionais) e negociação para avaliação das anotações (ao nível dos responsáveis dos processos).

A introdução da ferramenta MAPA em organizações reais revelou que pode ter um papel importante, não só na recolha das informações necessárias à atualização do modelo (para além do primeiro papel importante na validação do modelo construído inicialmente), mas também porque permitiu a abertura de um canal de comunicação, que fomenta a recolha e partilha de conhecimento sobre as atividades da organização. Permitiu ainda demonstrar que os atores podem desempenhar também o papel de modeladores ativos de forma colaborativa e distribuída.

A refinação do MACoP vai incidir na definição de categorias de anotação, com o objetivo de encontrar padrões de anotações com vista a melhorar a declaração de atualizações por parte dos atores organizacionais.

A ferramenta MAPA vai continuar a ser desenvolvida, agora com o objetivo central de a dotar com uma série de funcionalidades que potenciem a sua utilização frequente com a inclusão de ligação aos artefactos reais que são necessários durante a execução dos processos e atividades. Este objetivo vai proporcionar acesso aos manuais de qualidade em contexto e diretamente nos processos (por exemplo pode aceder-se às instruções de trabalho das atividades), acesso aos manuais de utilização dos sistemas informáticos necessários à execução de cada uma das atividades (no caso das atividades implicarem suporte informático) e acesso aos modelos dos documentos invocados nos processos. 


\section{Referências}

Adamides, E.D. \& Karacapilidis, N. (2006). A knowledge centred framework for collaborative business process modelling, Business Process Management Journal, 12(5), pp. $557-575$.

Becker-Kornstaedt, U. \& Reinert, R. (2002). A Concept to Support Process Model Maintenance through Systematic Experience Capture, Proceedings of the 14th international conference on Software engineering and knowledge engineering, July 15 -19, Ischia, Italy.

Bruno, G., Dengler, F., Jennings, B., Khalaf, R., Nurcan, S., Prilla, M, Sarini, M., Schmidt, R., \& Silva, R. (2011). Key challenges for enabling agile BPM with social software. Journal of Software Maintenance and Evolution: Research and Practice, 23(4), pp. 297-326.

Castela, N., Dias, P., Zacarias, M. and Tribolet, J.M. (2012). Collaborative maintenance of business process models, International Journal of Organisational Design and Engineering, 2(1), pp.61-84.

Ellis, C., Gibbs, S. \& Rein, G. (1991). Groupware: Some Issues and Experiences, Communications of the ACM, 34(1), pp. 38-58.

Gonzalez, P., Framinan, J. (2010). Tools for Collaborative Business Process Modeling, In Information Resources Management Association (Ed). Business Information Systems: Concepts, Methodologies, Tools and Applications (Vol. 3, Chap 10, pp. 636-648). USA:IDEA Group.

Magalhães, R. \& Tribolet, J. (2007). Engenharia Organizacional: das partes ao todo e do todo às partes na dialéctica entre pessoas e sistemas. In Costa, S.G., Vieira, L.M. \& Rodrigues, J.N. (Eds.). Ventos de Mudança (book chapter). Rio de Janeiro: Editora Fundo de Cultura.

Rittgen, P. (2007). Negotiating Models. In Krogstie, J, Opdahl, A. \& Sindre, G. (Eds.). Advanced Information Systems Engineering. (Vol. 4495, pp 561-573). (Lecture Notes in Computer Science). Berlin: Springer.

Schekkerman, J. (2006). How to Survive in the Jungle of Enterprise Architectures Frameworks (3rd Ed). Canada:Trafford Publishing.

Ssebuggwawo, D., Hoppenbrouwers, S., \& Proper, E. (2009). Interactions, goals and rules in a collaborative modelling session. In Persson, A \& Stirna, J. (Eds.). The Practice of Enterprise Modeling (Vol 39, pp. 54-68). (Lecture Notes in Business Information Processing). Berlin: Springer.

Tribolet J., Winter R. \& Caetano A. (2005). Editorial Message: Special Track on Organizational Engineering, Proceedings of the 2005 ACM symposium on Applied computing. Santa Fe, New Mexico, USA.

Tribolet, J. (2005). Organizações, Pessoas, Processos e Conhecimento: Da Reificação do Ser Humano como Componente do Conhecimento à "Consciência de Si" Organizacional. Sistemas de Informação Organizacionais (Chap. 16). Lisboa:Edições Sílabo 
Zacarias, M, Magalhães, R., Caetano, A., Pinto, H.S., \& Tribolet J. (2008). Towards organizational self-awareness: An initial architecture and ontology. In Rittgen, P. (Ed). Ontologies for Business Interactions (Chap.7, pp. 101-121). USA:IDEA Group. 